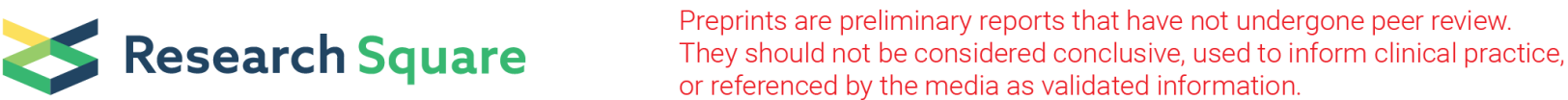

\section{SCM-198 Prevents Endometriosis By Reversing Low Autophagy Levels Of Endometriotic Stromal Cells Via Inhibiting TNF-A-Aromatase-Estrogen-Era Pathway And Promoting PR Expression}

\section{Yikong Lin}

Fudan University Shanghai Medical College

\section{Yunyun Li}

Fudan University Shanghai Medical College

Yue Li

Fudan University Shanghai Medical College

Dajin Li

Fudan University Shanghai Medical College

\section{Xiaolin Wang}

Macau University of Science and Technology

\section{Li Wang}

Fudan University Shanghai Medical College

\section{Min Yu}

Fudan University Shanghai Medical College

Yizhun Zhu

Macau University of Science and Technology

Jiajing Cheng

Tongji University Shanghai

Meirong Du ( $\nabla$ mrdu@fudan.edu.cn )

Fudan University Shanghai Medical College

\section{Research Article}

Keywords: SCM-198, EMS, TNF-a, aromatase, autophagy, apoptosis

Posted Date: January 11th, 2022

DOI: https://doi.org/10.21203/rs.3.rs-1228028/v1

License: (c) (i) This work is licensed under a Creative Commons Attribution 4.0 International License. Read Full License 
Page $2 / 21$ 


\section{Abstract}

Background: Endometriosis (EMS), an estrogen-dependent disease, is characterized by dysregulated inflammation and increased estrogen in ectopic lesions. However, the crosstalk and pathogenic mechanism of inflammation and estrogen has not been fully explored. SCM-198 is the synthetic form of leonurine with multiple pharmacological activities. Whether SCM-198 could inhibit the progress of EMS by regulating inflammation and estrogen signaling remains unknown.

Methods: The therapeutic effects and potential mechanisms of SCM-198 on EMS were analyzed by establishing EMS mice models and performing RNA-seq assay. ELISA was performed to detect estrogen and TNF- $a$ concentration in normal endometrial stromal cells (nESCs) and ectopic endometrial stromal cells (eESCs), with or without SCM-198 treatment. Western blotting, RNA silencing and plasmid overexpression were utilized to analyze the relationship among inflammation, endocrine and autophagy as well as the regulation of SCM-198 on inflammation-endocrine-autophagy axis.

Results: Increased estrogen-ERa signaling and decreased PR expression co-led to the hypo-autophagy state in eESCs, which further inhibited the apoptosis of eESCs. Highly expressed TNF-a in eESCs enhanced low-autophagy mediated anti-apoptosis effect by activating aromatase-estrogen-ERa signaling. SCM-198 inhibited the growth of ectopic lesions in EMS mouse model and promoted the apoptosis of eESCs both in vivo and in vitro. The apoptosis effect of SCM-198 on eESCs were realized by upregulating the autophagy level via inhibiting TNF- $\alpha$ activated aromatase-estrogen-ERa signaling and increasing PR expression.

Conclusion: Inflammation facilitated the progress of EMS by disturbing estrogen regulatory axis. SCM198 restrained the growth of EMS by regulating inflammation-endocrine-autophagy axis.

\section{Background}

Endometriosis (EMS) is defined as the presence of endometrial-like tissue outside the uterine cavity, with periodic bleeding of the ectopic lesions under the influence of ovarian hormones[1]. EMS is an estrogendependent and inflammation disordered disease that affects $10 \%$ women in reproductive age[2], and is associated with pelvic pain and infertility[3-6]. None of the proposed pathogenic theories can (retrograde menstruation, coelomic metaplasia and immune disorder theory) fully explain the origin and progression of EMS[7, 8]. Recent studies have pointed out that the aetiology of endometriosis is more related to the interaction between inflammation and endocrine $[9,10]$.

The treatment of EMS is tricky for its high recurrent rate ( 50\%)[11]. The surgical treatment is traumatic $[12,13]$ and medical therapy (e.g., progesterone, gonadotropin-releasing hormone (GnRH) agonists) is inefficient reducing the locally produced estrogen by ectopic lesions[11]. The expenditure of EMS is identified with the cost of diabetes, while the curative effect fails to meet the expectations. Therefore, it is urgent to comprehensively understand the pathogenesis of EMS and to find appropriate treatment. 
Enhanced survivability leads to continuous development of EMS[14]. Elevated estrogen level and resisted progesterone effect co-enhanced the survival of ectopic endometrium[15, 16]. Accumulated proinflammatory factors TNF-a enhanced the proliferation of ectopic endometrial stromal cells (eESCs) [17, 18]. Moreover, meta-analysis showed that TNF-a was associated with susceptibility to EMS and anti-TNFa therapy can relieve the pelvic pain associated with $\operatorname{EMS}[19,20]$. However, the specific crosstalk between endocrine and inflammation and its role in the occurrence and development of EMS needs to be further investigated.

Recently, the role of autophagy has been emphasized on the progress of EMS[21]. The autophagy level of eESCs is downregulated, which further promotes the survival and inhibits the apoptosis of eESCs[22-24]. Abnormal upregulated estrogen signaling has been found to inhibit autophagy in EMS[10,25]. Inflammation and autophagy can negatively regulate each other. Autophagy activation ameliorates inflammation[26, 27] and enhancing inflammation inhibits autophagy[28, 29]. Whether inflammation, endocrine and autophagy have regulatory networks to jointly mediate the process of EMS needs to be further explored.

SCM-198 is a synthetic form of leonurine[30, 31]. Studies have shown that SCM-198 has therapeutic effects on cardiovascular[32,33] and cerebrovascular disease[34]. Importantly, SCM-198 has been demonstrated to alleviate hyperalgesia in adenomyosis mice[35]. However, no study was reported to explore the effect of SCM-198 on EMS.

In the present study, we focused on the therapeutic effects of SCM-198 on EMS and explored the regulatory roles of SCM-198 in inflammation, endocrine and autophagy network of EMS. We found that SCM-198 obviously reversed the downregulated expression of PR and alleviated the higher production of TNF-a in eESCs. The activation of aromatase-estrogen-ERa signaling induced by augmented TNF-a was dramatically inhibited by SCM-198. Further, SCM-198 promoted the apoptosis of eESCs via reversing the inhibited autophagy level facilitated by activated TNF-a- aromatase-estrogen-ERa signaling and weakened PR expression. This study explained the complicated mechanisms involving immuneendocrine dysregulation of EMS and supplied a promising therapy for the refractory disease.

\section{Methods}

\section{Reagents and mice EMS model}

SCM-198 was kindly gifted by Dr Zhu Yizhun's lab. Female C57BL/6 mice (6-8 weeks old) were purchased from Shanghai JieSiJie Laboratory Animal Co. Ltd (shanghai., China). After two weeks of adaptation, mice were randomly selected as the donors of EMS model. Donor mice were intraperitoneally injected with $17 \beta$ estradiol (E2) (\#E2758, Sigma, St. Louis, USA) (0.2ug/g bodyweight) three times for one week. Vaginal smears were utilized to select estrus mice as the recipients of EMS mice model Mixture of the donor mouse uterine fragments and PBS were intraperitoneally injected to recipient mouse. Recipient mice were allowed to rest for one week. 
To investigate the effects of SCM-198 on EMS, recipient mice were randomly divided into three groups: EMS group, EMS + low-dose SCM-198 group (EMS+SCM-198 L, 7.5mg/kg) and EMS + high-dose SCM198 group (EMS+SCM-198 H, 15mg/kg). Intraperitoneally inject 200 $\mathrm{\mu l}$ of SCM-198 to the recipient mice according to the corresponding dose (once a day for a total one week). EMS group were given the same dose and frequency of PBS. One week later, the mice were sacrificed. Collect endometriotic tissue, uterus and peritoneal fluid for subsequent treatment.

\section{Collection of human samples and isolation of endometrial stromal cells}

Human ectopic endometrial tissues were obtained from 46 women with EMS (aged 22-45 years) via laparoscopy and human normal endometrial samples were collected from 10 healthy women (aged 2346 years) by uterine curettage at the Obstetrics and Gynecology Hospital of Fudan University. The endometrial tissues were minced (2-3-mm pieces) and digested in DMEM/F-12 containing collagenase type IV $\left(0.1 \%\right.$, Sigma, USA) for $30 \mathrm{~min}$ at $37^{\circ} \mathrm{C}$. The dispersed cells were then filtered through a 400 -mesh wire sieve to remove the undigested tissue pieces containing the glandular epithelium. After gentle centrifugation, the supernatant was discarded, and the cells were resuspended in DMEM/F-12 containing $10 \%$ fetal bovine serum (Gemini, Calabasas, USA), $100 \mathrm{IU} / \mathrm{ml}$ penicillin (Sigma, USA), $100 \mu \mathrm{g} / \mathrm{ml}$ streptomycin (Sigma, USA), and $1 \mathrm{\mu g} / \mathrm{ml}$ amphotericin B (Sangon, Shanghai, China) at $37^{\circ} \mathrm{C}$ in $5 \% \mathrm{CO}_{2}$.

\section{Immunohistochemical}

Placing the immunohistochemical sections at $60^{\circ} \mathrm{C}$ for $2 \mathrm{~h}$. Using xylene and gradient alcohol to Dewax and rehydrate the sections. It was incubated with $3 \%$ hydrogen peroxide and $5 \%$ bovine serum albumin successively to block endogenous peroxidase. Tissue sections were incubated with anti-mouse ERa (\#ab32063, Abcam, Cambridge, UK), PR (\#ab101688, Abcam, UK), overnight in a humid chamber at $4^{\circ} \mathrm{C}$. Wash the sections 3 times with PBS for 5 minutes each time. Cover the section with peroxidaseconjugated goat anti-rabbit or mouse IgG (\#GK500710, Gene Teck, San Francisco, USA) and incubate the secondary antibody for 30 minutes. Then it was reacted with 3,3-diaminobenzidine (DAB) and stained the nucleus with hematoxylin. Finally, the slices are dehydrated in gradient alcohol and xylene, and then the slices are mounted.

\section{Western blotting analysis}

The total proteins of endometrial stromal cells (ESCs), mouse uterine and ectopic lesions were extracted using radioimmunoprecipitation (RIPA) assay buffer (Beyotime, Shanghai, China) supplemented with protease and phosphatase inhibitors (Sigma, USA). The protein concentration was measured using a BCA protein assay kit (Beyotime, China). After denaturation, equal amounts of protein were separated via SDSpolyacrylamide gel electrophoresis (PAGE) before wet transfer onto polyvinylidene difluoride membranes. Nonspecific binding sites were blocked by incubating the membranes with $5 \%$ skim milk in Tris-buffered saline with $0.1 \%$ Tween 20 (TBS-T) for $1 \mathrm{~h}$. Then, the membranes were incubated overnight at $4^{\circ} \mathrm{C}$ with primary antibodies (1:1000) against aromatase(\#14528, CST, Boston, USA), ERa (\#ab32063, Abcam, UK), PR(\#ab32085, Abcam, UK), LC3B(\#3868, CST, USA), BECN1 (\#ab207612, Abcam, UK), Bcl-2(\#2870, CST, 
USA), Bax(\#12105, CST, USA), FN1 (\#ab2413, Abcam, UK), vimentin(\#5741, CST, USA), a-tubulin(\#ab7291, Abcam, UK) and GAPDH (\#10112, Arigo, Taiwan, China). Subsequently, membranes were incubated with appropriate horseradish peroxidase-conjugated anti-rabbit (\#65351, Arigo, China) or anti-mouse (\#65350, Arigo, China) IgG secondary antibodies for $1 \mathrm{~h}$ at room temperature. After three washes with TBS-T, immunopositive bands on the blots were visualized using chemiluminescent HRP substrate (\#WBKLS0100, Millipore, Boston, USA) on the enhanced chemiluminescence detection system (Merck Millipore, USA).

\section{RNA-Seq Data Analysis}

Differential expression was computed with DESeq2, and the corrected gene or transcriptome expression value, the corrected fold-change, and the p-value and FDR value of the significance of the difference can be obtained. Set $p$-value $<0.05$ and Fold change greater than 1.2 times or less than 0.83333 times as difference, then we get 1616 differential genes. Based on the differentially expressed genes between the different groups, the GO database and the KEGG database were used for functional enrichment and pathway enrichment, respectively. Statistical analysis was performed in the R version 4.0.4 according to $\mathrm{p}$-value $<0.05$. Bubble charts and volcano plot were performed by using ggplot and cluster profiler packages.

\section{Quantitative real-time PCR}

Total RNA was extracted using TRIzol reagent (Invitrogen, California, USA) and then reverse-transcribed into first-strand complementary DNA (cDNA) (Takara, Kyoto, Japan) according to the manufacturer's instructions. The synthesized cDNA was amplified with specific primers and SYBR Green (Takara, Japan) using an ABI PRISM 7900 Sequence Detection System (Applied Biosystems, California, USA). Triplicate samples were examined for each condition. A comparative threshold cycle value was normalized for each sample using the $2^{-\Delta \Delta} \mathrm{Ct}$ method.

\section{ELISA}

The supernatant of normal endometrial stromal cells ( $\mathrm{nESCs}$ ) and differently treated eESCs was harvested and assayed by ELISA according to the manufacturer's instructions (estrogen, \#CSB-E07286h, CUSABIO, Shanghai, China; TNF-a, \#BDEL-0049, Biodragon, Beijing, China), to detect the levels of secreted estrogen and TNF-a.

\section{Plasmid Overexpression and siRNA transfection}

Aromatase overexpression (Aromatase ${ }^{\text {over}}$ ) plasmid and negative control plasmid were purchased from Shanghai Genechem Co., LTD. (shanghai, China). Aromatase siRNA (siAromatase) and control siRNA were purchased from Shanghai Genepharma Co., LTD. (shanghai, China). Aromatase overexpressed plasmid and negative control plasmid (Ctrl) were transfected into eESCs cells by liposome transient transfection when the fusion degree reached about 70-80\% in 6-well plate. Transfected cells were 
incubated for $24 \mathrm{~h}$ at $37^{\circ} \mathrm{C}$ and then collected for further study. The transfection process of siAromatase were similar to the overexpressed aromatase transfection.

\section{Statistical analysis}

Prism 8 software (GraphPad) was used for data analysis. Statistical significance was determined using Student's t-test for 2-group or one-way ANOVA for multiple group comparisons. The data are presented as mean $\pm S D$. Statistical significance was attained when $P<0.05$.

\section{Results}

\section{SCM-198 suppresses the endometriotic growth both in vivo and in vitro}

We first explored whether SCM-198 could alleviate the development of EMS by using mice EMS model. Fig. 1A showed the general process of establishing EMS mice model. As shown, SCM-198 not only decreased the weight and size of mice ectopic lesions (Fig. 1B, C), but also reduced the wall thickness of EMS lesions (Fig. 1D). In addition, masson staining showed that SCM-198 treatment significantly reduced collagen accumulation in ectopic lesions (Fig. 1E). Western blotting results revealed that SCM-198 inhibited the expression of anti-apoptosis protein $\mathrm{Bcl}-2$ and promoted the expression of pro-apoptosis protein Bax in ectopic lesions at both low and high administration (Fig. 1F). Consistent with in vivo results, in vitro analysis showed that SCM-198 inhibited Bcl-2 and promoted Bax expression in eESCs (Fig. 1G). Meanwhile, fibrosis related molecules fibronectin 1 (FN1) and vimentin were also decreased in SCM-198 dose-dependently treated eESCs (Fig. 1H). These results suggest that SCM-198 has the ability to accelerate the apoptosis and attenuate the growth and fibrosis of EMS both in vivo and in vitro.

\section{SCM-198 promotes the autophagy level and inhibits estrogen signaling of EMS}

To investigate the under mechanism of SCM-198 in restraining EMS, we performed RNA-seq in SCM-198 treated and untreated ectopic lesions of EMS mice model. We observed a total of 1616 differentially expressed genes, with 701 genes being upregulated and 915 genes being downregulated in SCM-198 treated ectopic lesions compared with that in EMS groups (Fig. 2A). GO enrichment and KEGG pathway analysis revealed that SCM-198 downregulated the autophagy inhibitor molecules and estrogen receptor pathway of ectopic lesions (Fig. 2B, C).

To confirm the results from bioinformatics analysis, we first analyzed the autophagy related gene expression in ectopic lesions. The results in Fig. 2D showed that SCM-198 could extensively promote the mRNA expression of autophagy positively-related proteins in ectopic lesions, such as Map1lc3b, Becn1Ulk1, Atg3, Atg4b, Atg5, Atg7, Gabarap, Atg9a and Atg10. Further, western blotting results confirmed that SCM-198 could promote the autophagy level by upregulating the ratio of autophagy-positively related proteins LC3B-II/ $/$ and BECN1 expression (Fig. 2E). Meanwhile, SCM-198 reversed the imbalanced expression of ERa and PR in EMS ectopic lesions by up-regulating PR and down-regulating ERa 
expressions (Fig. 2F, G). These results suggest that SCM-198 could promote the autophagy level and inhibit estrogen signaling of EMS.

\section{The ERa/PR imbalance contributes to the hypo-autophagy state of eESCs.}

We then assessed estrogen, hormone receptor, and autophagy level of eESCs in EMS patients. Higher production of estrogen (Fig. 3A) and upregulated ERa (Fig. 3B) were observed in eESCs. Compared with nESCs, LC3B-II/ $\triangle$ and BECN1 were downregulated in eESCs, suggesting a lower autophagy level in eESCs (Fig. 3C). To explore the relationship between estrogen signaling and autophagy, eESCs were treated with E2. The results showed that E2 treatment dose dependently increased ERa expression and inhibited

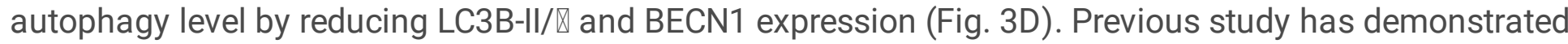
that ERa inhibited autophagy in eESCs[10]. Thus, locally produced high level of estrogen led to increased ERa, which further inhibited autophagy. Meanwhile, PR expression was decreased in eESCs (Fig. 3E). Progesterone increased PR expression and promoted autophagy in a dose-dependent manner (Fig. 3F).

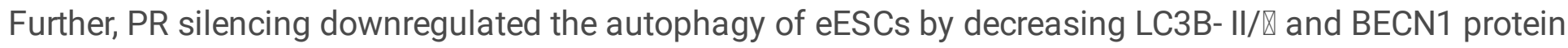
expression, implying that decreased PR contributed to the hypo-autophagy state in eESCs (Fig. 3G). Together, these results suggest that local high estrogen level leads to increased ERa level and the imbalanced of ERa/PR expression in ectopic lesions promotes the hypo-autophagy in eESC.

\section{SCM-198 promotes autophagy mediated apoptosis of eESC by inhibiting estrogen-ERa pathway and promoting PR expression}

To investigate whether SCM-198 promotes autophagy level by inhibiting estrogen signaling, eESCs were treated with SCM-198. The results showed that SCM-198 dose dependently downregulated estrogen level, ERa expression (Fig. 4A, B) and enhanced autophagy by upregulating LC3B-II/I and BECN1 of eESCs (Fig. 4B). Importantly, augmented ERa expression and inhibited autophagy induced by E2 were reversed by SCM-198 administration in eESCs (Fig. 4C). Meanwhile, SCM-198 dose-dependently upregulated PR expression (Fig. 4D) and reversed the inhibited autophagy mediated by PR silencing (Fig. 4E). Further, by using autophagy inhibitor 3-MA, we demonstrated that low autophagy level was conducive to cell antiapoptosis (Fig. 4F). And SCM-198 exerted pro-apoptosis effects on eESCs via promoting autophagy (Fig. $4 \mathrm{~F})$.

Together, these results imply that SCM-198 could promote the apoptosis of eESCs by enhancing autophagy via inhibiting estrogen pathway and promoting PR expression.

\section{TNF-a positively regulates aromatase-estrogen-ERa pathway in eESCs}

Locally disordered inflammatory factors and increased estrogen both promoted the growth and proliferation of EMS ectopic lesions. To investigate the crosstalk between inflammation and estrogen signaling pathway in EMS, we first detected the level of pro-inflammation cytokine TNF-a and the expression of aromatase (key enzyme of estrogen production) in eESCs. As shown, the mRNA expression and concentration of TNF-a was significantly increased (Fig. 5A) and the expression of aromatase was 
also upregulated in eESCs (Fig. 5B). Next, eESCs were treated with TNF-a or R-7050, a tumor necrosis factor receptor (TNFR) antagonist. TNF- $a$ obviously promoted estrogen signaling pathway by increasing the expressions of aromatase and ERa (Fig. 5C), and elevating estrogen concentration (Fig. 5D). Aromatase silencing decreased estrogen level and ERa expression (Fig. 5E, F). Aromatase overexpression increased estrogen level and ERa expression (Fig. 5G, H), indicating that aromatase-estrogen signaling positively regulate ERa. Further, to demonstrate TNF-a positively regulate estrogen-ERa signaling through aromatase, aromatase was silenced under the treatment of TNF-a. As shown in Fig. $5 \mathrm{I}$ and $\mathrm{J}$, aromatase silencing abolished the promotive effect of TNF-a on estrogen production and ERa expression. These data indicate that TNF-a can positively regulate the aromatase-estrogen-ERa pathway and inflammatory disorders can promote estrogen production to accelerate the development of EMS.

\section{The pro-apoptotic effects of SCM-198 are realized by down-regulating aromatase-estrogen pathway via inhibiting TNF-a}

To explore whether the promotive effects of SCM-198 on autophagy are realized by inhibiting TNF-aaromatase-estrogen-ERa pathway, we treated eESCs with different concentrations of SCM-198. SCM-198 dose dependently decreased the concentrations of TNF-a (Fig. 6A) and estrogen (Fig. 4A), and suppressed the expression of aromatase and ERa in eESCs (Fig. 6B). Furthermore, the anti-apoptosis effect by TNF-a-aromatase-estrogen-ERa signaling mediated low autophagy could be reversed by SCM198 administration (Fig. 6C, D). These results suggest that inflammation suppresses autophagy via estrogen signaling, thereby inhibiting the apoptosis of eESCs. SCM-198 could downregulate TNF-aaromatase-estrogen-ERa pathway, eventually promoting autophagy and ultimately accelerating the apoptosis of eESCs (Fig. 7).

\section{Discussion}

EMS, a common obstetrics and gynecology diseases, is characterized by the growth of endometrial tissue appears outside the uterine cavity. Owing to the severe complications (pelvic pain and infertility) and high prevalence $(\sim 10 \%)$, multiple therapies have been proposed to deal with the disease[36, 37]. However, still up to $50 \%$ recurrence rate has been observed in EMS patients, because neither medication nor surgical treatment is effective in eradicating the growth of ectopic lesions[11]. Finding and identifying effective medication for the treatment of EMS is beneficial for improving the quality of women life and relieving substantial economic burden. SCM-198, a synthetic form of leonurine, proved to have pain relief pharmacological effects on adenomyosis[35]. In the present study, we first identified the therapeutic effects of SCM-198 on EMS, as evidenced by decreased weight and size, reduced collagen accumulation and augmented $\mathrm{Bax} / \mathrm{Bcl}-2$ ratio of mice ectopic lesions.

Aberrant autophagy has stepped into the spotlight on the stage of EMS pathogenesis[21, 38]. Cumulative studies have uncovered that the autophagy level of ectopic endometrium is decreased[39, 40]. More important, low-autophagy level contributes to the survival of ectopic endometrium, as corroborated by enhanced apoptosis and decreased proliferation of eESCs under the induction of autophagy[24, 41]. 
Then, we inquired whether autophagy is involved in the mechanisms of SCM-198 on treating EMS. RNASeq results based on ectopic foci suggested that SCM-198 treatment significantly promoted autophagy of eESCs. The upregulated mRNA levels of autophagy related genes in ectopic lesions and increased LC3B and BECN1 expression both in vitro and in vivo under SCM-198 treatment further confirmed the promotive effects of SCM-198 on autophagy level of eESCs.

The imbalanced endocrine microenvironment of ectopic endometrium, manifested with high estrogen signaling and progesterone resistance, have inescapable responsibility for the growth of ectopic endometrium. Increased estrogen production supported by elevated aromatase expression potentiates the proliferation of ectopic lesion[42-44], which could be reinforced by resisted progesterone signaling mediated by decreased PR expression[45, 46]. Multiple studies have emphasized that inhibiting autophagy is an important pathway for estrogen to restrain the apoptosis and facilitate growth of ectopic lesions $[10,25]$. Whether the promotive effects of SCM-198 on autophagy are realized by repairing the damaged hormonal endocrine networks. We verified that increased estrogen signaling and impaired progesterone signaling co-drive the decline of autophagy in eESCs. In consistent with the hypothesis, the upregulation effects of SCM-198 on autophagy are mediated by increasing PR expression and decreasing aromatase-estrogen-ERa signaling in eESCs.

The current consensus is that dysregulated pelvic inflammatory process plays a crucial role in the initiation and progression of EMS[47]. Abnormally upregulated pro-inflammatory cytokine TNF- $\alpha$ is closely related to the pelvic pain and infertility associated with $\operatorname{EMS}[20,48]$. Our data confirmed that there was an obviously upregulation in both mRNA expression and concentration of TNF-a. The upraised production of TNF-a could be suppressed the treatment of SCM-198, which has been identified as an antiinflammation drug. More important, we demonstrated that elevated TNF- $a$ augmented the activation of estrogen-ERa signaling by increasing the expression of aromatase. Further, TNF- $a$ inhibited the autophagy of eESCs by promoting estrogen signaling, suggesting inflammation-endocrine-autophagy axis plays an indispensable role in ectopic endometrial survival. Notably, we found that SCM-198 abated TNF-a induced estrogen signaling activation and autophagy inhibition, ultimately restrained the apoptosis of eESCs.

\section{Conclusions}

In summary, the disturbed inflammation, endocrine and autophagy made EMS to be complicated and difficult to cure. Elevated levels of inflammation, imbalanced estrogen and progesterone and inactivated autophagy co-contribute to the progression of EMS. SCM-198 focused on rectifying the aberrant inflammation-endocrine-autophagy axis. SCM-198 reversed the low autophagy level of eESCs via inhibiting TNF-a-aromatase-estrogen-ERa pathway and promoting PR expression, and ultimately alleviated the development of EMS. To sum up, this study provided a theoretical basis for the potential application of SCM-198 on the treatment of EMS.

\section{Abbreviations}


EMS: endometriosis; eESCs: endometrial stromal cells; nESCs: normal endometrial stromal cells; eESCs: ectopic endometrial stromal cells; GnRH: gonadotropin-releasing hormone; E2: 17 $\beta$-estradiol; RIPA: radioimmunoprecipitation; $C D N A$ : complementary DNA; DAB: diaminobenzidine; FN1: fibrosis related molecules fibronectin 1; TNFR: tumor necrosis factor receptor.

\section{Declarations}

\section{Ethics approval and consent to participate}

Our research was approved by the Human Research Ethics Committee of the Obstetrics and Gynecology Hospital of Fudan University and all experiments were performed in accordance with relevant guidelines and regulations (No. Kyy2016-4) (Shanghai, China).

\section{Consent for publication}

All authors have read the manuscript and approved the final version.

\section{Availability of data and materials}

The datasets used and/or analyzed during the current study are available from the corresponding author on reasonable request.

\section{Competing interests}

The authors declare no competing interests.

\section{Founding}

This research was supported by the grants from the National Basic Research Program of China (2021YFE0206500), the National Natural Science Foundation of China (31970859, 81630036, 91542116), the international cooperation project between Macau and Shanghai (20410760300), Strategic Collaborative Research Program of the Ferring Institute of Reproductive Medicine (FIRMA200504), Innovation-oriented Science and Technology Grant from NHC Key Laboratory of Reproduction Regulation (CX2017-2), and Innovative research team of high-level local universities in Shanghai and a key laboratory program of the Education Commission of Shanghai Municipality (ZDSYS14005).

\section{Authors' contributions}

Yikong Lin and Yunyun Li designed and performed the experiments, and drafted the manuscript; Yue Li performed literature searches, data analysis and revised the manuscript; Li Wang, Dajin Li, Xiaolin Wang performed data interpretation and revised the manuscript. Meirong Du Jiajing Cheng and Yizhun Zhu conceived the project, analyzed the data and revised the manuscript. All authors reviewed the manuscript, and approved the final manuscript. 
Acknowledgements

Not applicable.

\section{References}

1. Chapron C, Marcellin L, Borghese B, Santulli P. Rethinking mechanisms, diagnosis and management of endometriosis. Nat Rev Endocrinol. 2019;15:666-682.

2. Shafrir AL, Farland LV, Shah DK, Harris HR, Kvaskoff M, Zondervan K, et al. Risk for and consequences of endometriosis: A critical epidemiologic review. Best Pract Res Clin Obstet Gynaecol. 2018;51:1-15.

3. Carvalho N, Margatho D, Cursino K, Benetti-Pinto CL, Bahamondes L. Control of endometriosisassociated pain with etonogestrel-releasing contraceptive implant and 52-mg levonorgestrel-releasing intrauterine system: randomized clinical trial. Fertil Steril. 2018;110:1129-1136.

4. Lai ZZ, Yang HL, Ha SY, Chang KK, Mei J, Zhou WJ, et al. Cyclooxygenase-2 in Endometriosis. Int J Biol Sci. 2019;15:2783-2797.

5. Lai ZZ, Yang HL, Shi JW, Shen HH, Wang Y, Chang KK, et al. Protopanaxadiol improves endometriosis associated infertility and miscarriage in sex hormones receptors-dependent and independent manners. Int J Biol Sci. 2021;17:1878-1894.

6. Nezhat C, Vang N, Tanaka PP, Nezhat C. Optimal Management of Endometriosis and Pain. Obstet Gynecol. 2019;134:834-839.

7. Vercellini P, Viganò P, Somigliana E, Fedele L. Endometriosis: pathogenesis and treatment. Nat Rev Endocrinol. 2014;10:261-275.

8. Burney RO, Giudice LC. Pathogenesis and pathophysiology of endometriosis. Fertil Steril. 2012;98:511519.

9. Patel BG, Lenk EE, Lebovic DI, Shu Y, Yu J, Taylor RN. Pathogenesis of endometriosis: Interaction between Endocrine and inflammatory pathways. Best Pract Res Clin Obstet Gynaecol. 2018;50:50-60.

10. Zhang B, Zhou WJ, Gu CJ, Wu K, Yang HL, Mei J, et al. The ginsenoside PPD exerts anti-endometriosis effects by suppressing estrogen receptor-mediated inhibition of endometrial stromal cell autophagy and NK cell cytotoxicity. Cell Death Dis. 2018;9:574.

11. Becker CM, Gattrell WT, Gude K, Singh SS. Reevaluating response and failure of medical treatment of endometriosis: a systematic review. Fertil Steril. 2017;108:125-136.

12. Zakhari A, Edwards D, Ryu M, Matelski JJ, Bougie O, Murji A. Dienogest and the Risk of Endometriosis Recurrence Following Surgery: A Systematic Review and Meta-analysis. J Minim Invasive Gynecol. 
13. Guo SW. Recurrence of endometriosis and its control. Hum Reprod Update. 2009;15:441-461.

14. Liu Y, Li M, Wei C, Tang L, Sheng Y, Liu Y, et al. TSP1-CD47-SIRPa signaling facilitates the development of endometriosis by mediating the survival of ectopic endometrium. Am J Reprod Immunol. 2020;83:e13236.

15. Marquardt RM, Kim TH, Shin JH, Jeong JW. Progesterone and Estrogen Signaling in the Endometrium: What Goes Wrong in Endometriosis? Int J Mol Sci. 2019;20.

16. Kim TH, Young SL, Sasaki T, Deaton JL, Schammel DP, Palomino AW, et al. Role of SIRT1 and Progesterone Resistance in Normal and Abnormal Endometrium. J Clin Endocrinol Metab. 2021.

17. Braun DP, Ding J, Dmowski WP. Peritoneal fluid-mediated enhancement of eutopic and ectopic endometrial cell proliferation is dependent on tumor necrosis factor-alpha in women with endometriosis. Fertil Steril. 2002;78:727-732.

18. Grandi G, Mueller M, Bersinger N, Papadia A, Nirgianakis K, Cagnacci A, et al. Progestin suppressed inflammation and cell viability of tumor necrosis factor-a-stimulated endometriotic stromal cells. Am J Reprod Immunol. 2016;76:292-298.

19. Cao XL, Chai J, Yu YY, Tian X, Zhao JY, Yu LY, et al. Association of TNF-a gene T-1031C polymorphism with endometriosis: A meta-analysis. Am J Reprod Immunol. 2020;84:e13305.

20. Lu D, Song H, Shi G. Anti-TNF-a treatment for pelvic pain associated with endometriosis. Cochrane Database Syst Rev. 2013:Cd008088.

21. He R, Liu X, Zhang J, Wang Z, Wang W, Fu L, et al. NLRC5 Inhibits Inflammation of Secretory Phase Ectopic Endometrial Stromal Cells by Up-Regulating Autophagy in Ovarian Endometriosis. Front Pharmacol. 2020;11:1281.

22. Choi J, Jo M, Lee E, Oh YK, Choi D. The role of autophagy in human endometrium. Biol Reprod. 2012;86:70.

23. Li M, Lu MS, Liu ML, Deng S, Tang XH, Han C, et al. An Observation of the Role of Autophagy in Patients with Endometriosis of Different Stages during Secretory Phase and Proliferative Phase. Curr Gene Ther. 2018;18:286-295.

24. Choi J, Jo M, Lee E, Kim HJ, Choi D. Differential induction of autophagy by mTOR is associated with abnormal apoptosis in ovarian endometriotic cysts. Mol Hum Reprod. 2014;20:309-317.

25. Mei J, Zhu XY, Jin LP, Duan ZL, Li DJ, Li MQ. Estrogen promotes the survival of human secretory phase endometrial stromal cells via CXCL12/CXCR4 up-regulation-mediated autophagy inhibition. Hum 
Reprod. 2015;30:1677-1689.

26. Cosin-Roger J, Simmen S, Melhem H, Atrott K, Frey-Wagner I, Hausmann M, et al. Hypoxia ameliorates intestinal inflammation through NLRP3/mTOR downregulation and autophagy activation. Nat Commun. 2017;8:98.

27. Zhang S, Hu L, Jiang J, Li H, Wu Q, Ooi K, et al. HMGB1/RAGE axis mediates stress-induced RVLM neuroinflammation in mice via impairing mitophagy flux in microglia. J Neuroinflammation. 2020;17:15.

28. Espín-Palazón R, Martínez-López A, Roca FJ, López-Muñoz A, Tyrkalska SD, Candel S, et al. TNFa Impairs Rhabdoviral Clearance by Inhibiting the Host Autophagic Antiviral Response. PLoS Pathog. 2016;12:e1005699.

29. Hong X, Min SN, Zhang YY, Lin YT, Wang F, Huang Y, et al. TNF-a Suppresses Autophagic Flux in Acinar Cells in IgG4-Related Sialadenitis. J Dent Res. 2019;98:1386-1396.

30. Zhu YZ, Wu W, Zhu Q, Liu X. Discovery of Leonuri and therapeutical applications: From bench to bedside. Pharmacol Ther. 2018;188:26-35.

31. Li YY, Lin YK, Liu XH, Wang L, Yu M, Li DJ, et al. Leonurine: From Gynecologic Medicine to Pleiotropic Agent. Chin J Integr Med. 2020;26:152-160.

32. Liu C, Guo W, Shi X, Kaium MA, Gu X, Zhu YZ. Leonurine-cysteine analog conjugates as a new class of multifunctional anti-myocardial ischemia agent. Eur J Med Chem. 2011;46:3996-4009.

33. Liu XH, Pan LL, Deng HY, Xiong QH, Wu D, Huang GY, et al. Leonurine (SCM-198) attenuates myocardial fibrotic response via inhibition of NADPH oxidase 4. Free Radic Biol Med. 2013;54:93-104.

34. Xie YZ, Zhang XJ, Zhang C, Yang Y, He JN, Chen YX. Protective effects of leonurine against ischemic stroke in mice by activating nuclear factor erythroid 2-related factor 2 pathway. CNS Neurosci Ther. 2019;25:1006-1017.

35. Nie J, Liu X. Leonurine Attenuates Hyperalgesia in Mice with Induced Adenomyosis. Med Sci Monit. 2017;23:1701-1706.

36. Bulun SE, Yilmaz BD, Sison C, Miyazaki K, Bernardi L, Liu S, et al. Endometriosis. Endocr Rev. 2019;40:1048-1079.

37. Zondervan KT, Becker CM, Missmer SA. Endometriosis. N Engl J Med. 2020;382:1244-1256.

38. Yang HL, Mei J, Chang KK, Zhou WJ, Huang LQ, Li MQ. Autophagy in endometriosis. Am J Transl Res. 2017;9:4707-4725.

39. Siracusa R, D'Amico R, Impellizzeri D, Cordaro M, Peritore AF, Gugliandolo E, et al. Autophagy and Mitophagy Promotion in a Rat Model of Endometriosis. Int J Mol Sci. 2021;22. 
40. Mei J, Zhou WJ, Zhu XY, Lu H, Wu K, Yang HL, et al. Suppression of autophagy and HCK signaling promotes PTGS2(high) FCGR3(-) NK cell differentiation triggered by ectopic endometrial stromal cells. Autophagy. 2018;14:1376-1397.

41. Choi J, Jo M, Lee E, Lee DY, Choi D. Dienogest enhances autophagy induction in endometriotic cells by impairing activation of AKT, ERK1/2, and mTOR. Fertil Steril. 2015;104:655-664.e651.

42. Machado DE, Alessandra-Perini J, Menezes de Mendonça E, Claudino MC, Nasciutti LE, Sola-Penna M, et al. Clotrimazole reduces endometriosis and the estrogen concentration by downregulating aromatase. Reproduction. 2020;159:779-786.

43. Suganuma I, Mori T, Ito F, Tanaka Y, Sasaki A, Matsuo S, et al. Peroxisome proliferator-activated receptor gamma, coactivator $1 \mathrm{a}$ enhances local estrogen biosynthesis by stimulating aromatase activity in endometriosis. J Clin Endocrinol Metab. 2014;99:E1191-1198.

44. Mori T, Ito F, Koshiba A, Kataoka H, Tanaka Y, Okimura H, et al. Aromatase as a target for treating endometriosis. J Obstet Gynaecol Res. 2018;44:1673-1681.

45. Eaton JL, Unno K, Caraveo M, Lu Z, Kim JJ. Increased AKT or MEK1/2 activity influences progesterone receptor levels and localization in endometriosis. J Clin Endocrinol Metab. 2013;98:E18711879.

46. Reis FM, Coutinho LM, Vannuccini S, Batteux F, Chapron C, Petraglia F. Progesterone receptor ligands for the treatment of endometriosis: the mechanisms behind therapeutic success and failure. Hum Reprod Update. 2020;26:565-585.

47. Samimi M, Pourhanifeh MH, Mehdizadehkashi A, Eftekhar T, Asemi Z. The role of inflammation, oxidative stress, angiogenesis, and apoptosis in the pathophysiology of endometriosis: Basic science and new insights based on gene expression. J Cell Physiol. 2019;234:19384-19392.

48. Wang XM, Ma ZY, Song N. Inflammatory cytokines IL-6, IL-10, IL-13, TNF-a and peritoneal fluid flora were associated with infertility in patients with endometriosis. Eur Rev Med Pharmacol Sci. 2018;22:25132518.

\section{Figures}


A

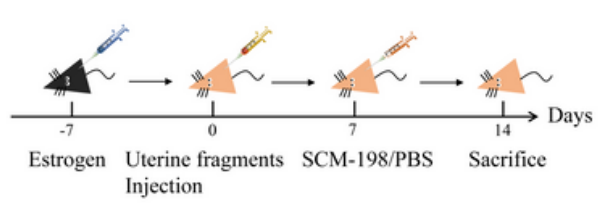

B

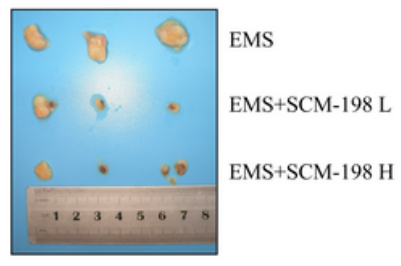

D

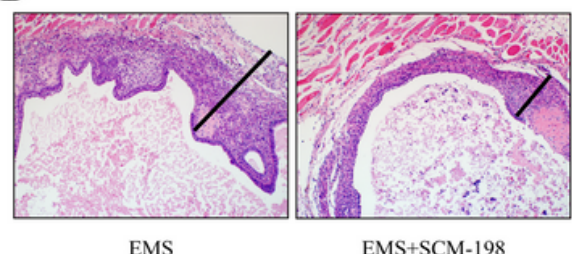

G

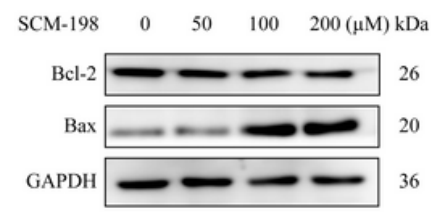

E

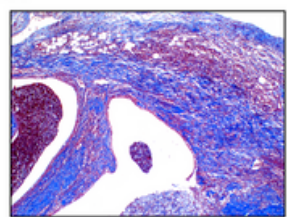

EMS

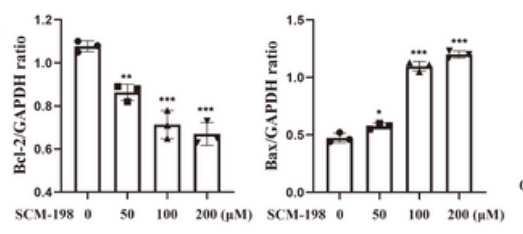

$\mathrm{C}$

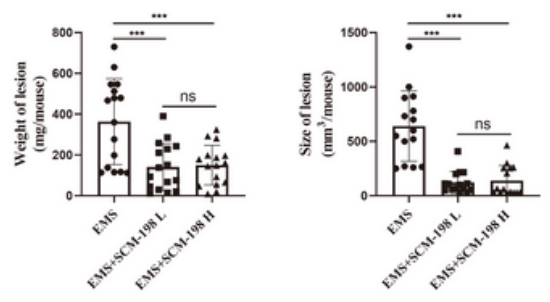

$\mathrm{F}$

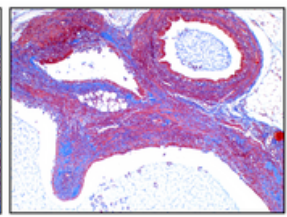

EMS+SCM-198

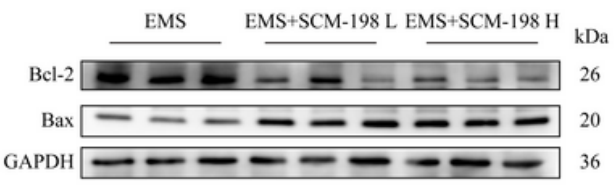

$\mathrm{H}$
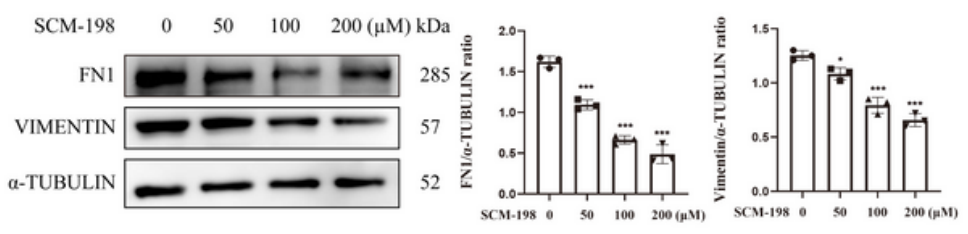

Figure 1

SCM-198 inhibits the endometriotic growth both in vivo and in vitro.

EMS mice were treated with or without SCM-198 at low (EMS+SCM-198 L, 7.5mg/kg) or high (EMS+SCM$198 \mathrm{H}, 15 \mathrm{mg} / \mathrm{kg}$ ) dose once a day for one week. (A) The flow chart of establishing mice EMS model. (B) Representative images of the ectopic lesions from EMS mice. (C) Quantitative results for the weight $(n=16)$ and size $(n=15)$ of ectopic lesions from EMS mice. (D) Thickness of ectopic cyst wall from EMS mice. (E) Masson staining was performed to detect the collagen fibers of ectopic lesions. (F) Western blotting was utilized to analyze the protein levels of $\mathrm{Bcl}-2$ and Bax in ectopic lesions. $(\mathbf{G}, \mathbf{H})$ The eESCs were treated with different doses of SCM-198 for 48h, then western blotting was utilized to analyze the protein levels of $\mathrm{Bcl}-2, \mathrm{Bax}, \mathrm{FN} 1$, vimentin $(\mathrm{n}=3)$. Data are the presented as the mean $\pm \mathrm{SD}\left({ }^{*} P<0.05, \star \star P\right.$ $<0.01$, and ${ }^{\star *} P<0.001$; ns, not significant). 
A

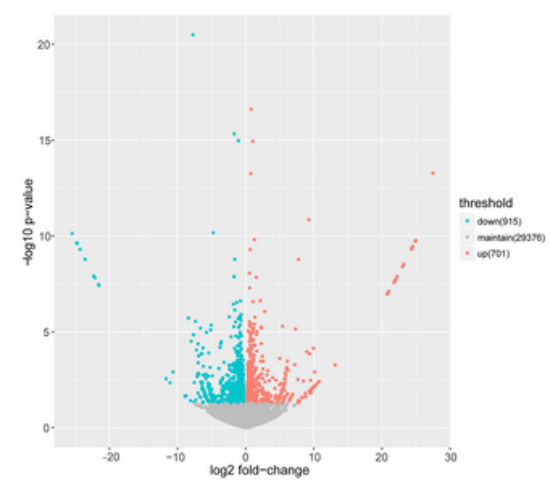

$\mathrm{D}$
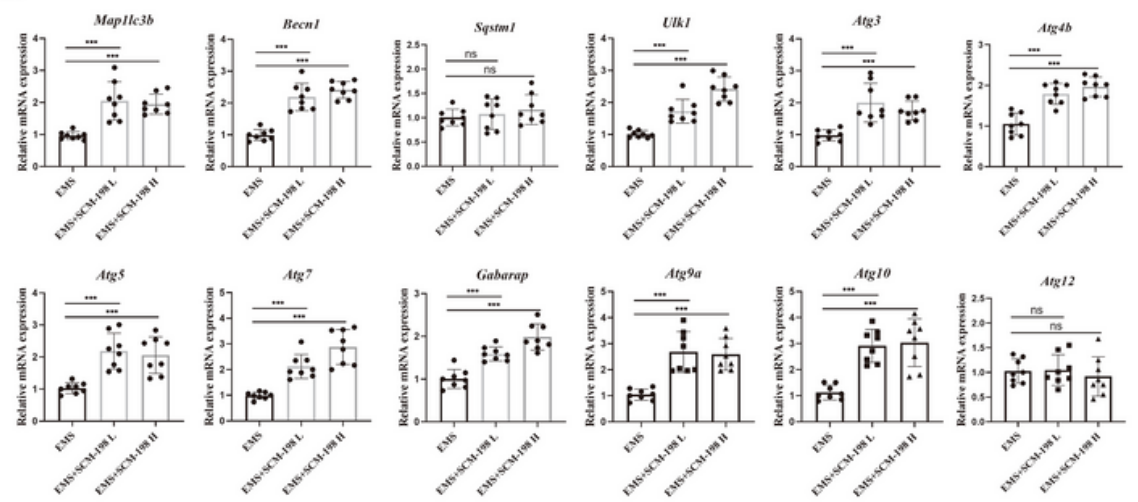

$\mathrm{C}$
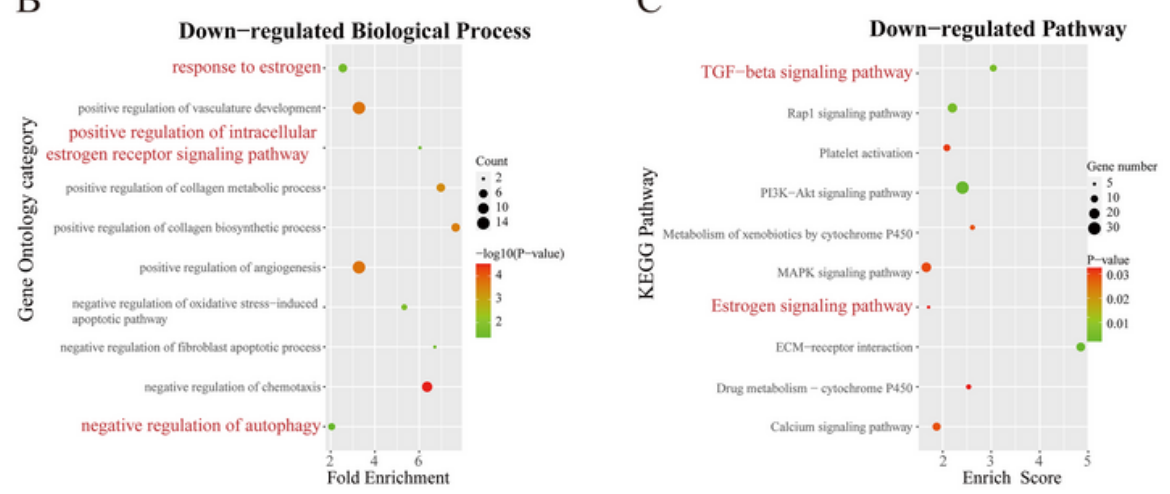

E

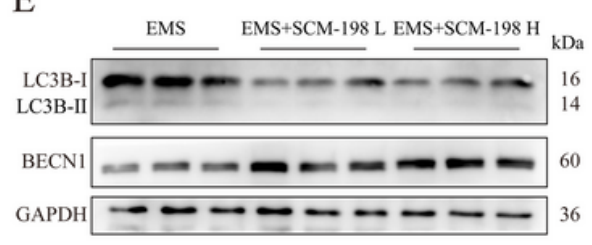

$\mathrm{F}$
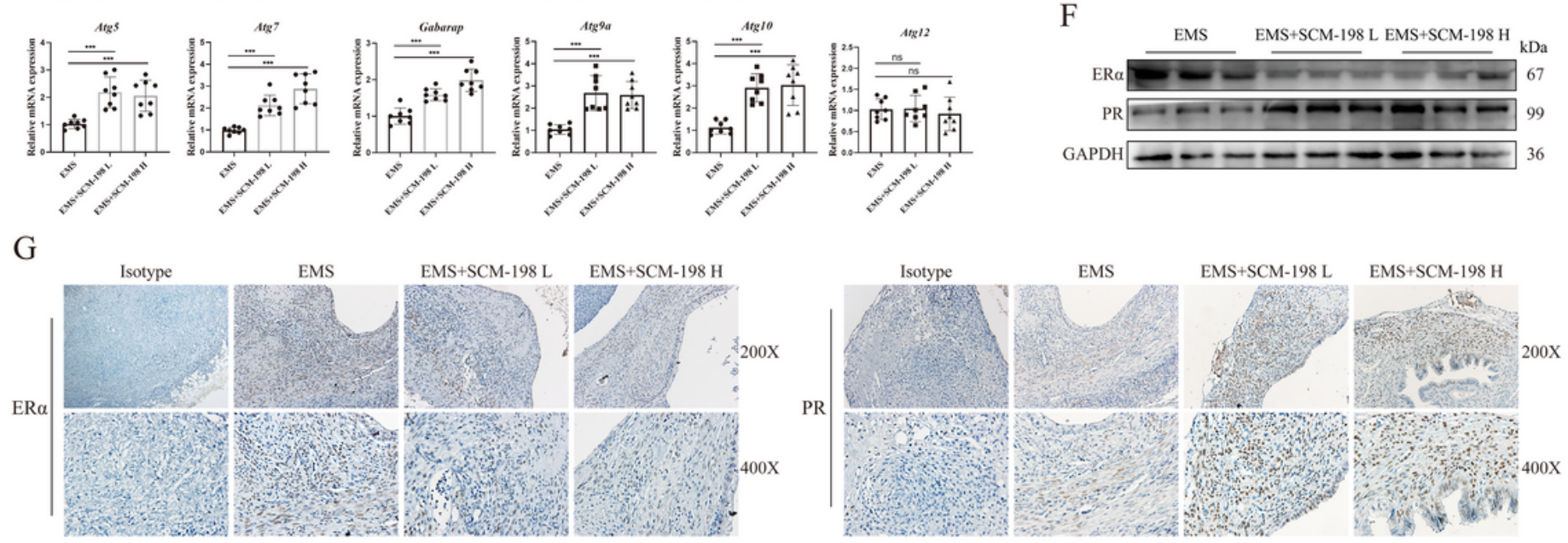

Figure 2

SCM-198 promotes the autophagy and inhibits estrogen signaling in EMS mouse model.

(A) Volcano plot of differentially expressed genes of ectopic lesions in SCM-198-treated EMS mice compared with that from the untreated EMS mice. The x-axis showed Log2 (Fold Change) in expression, and the $y$-axis showed the -Log10 ( $p$-value) of gene being expressed differentially (blue: downregulated genes; red: upregulated genes). (B) GO enrichment of biological process for the downregulated genes. (C) KEGG pathway analysis for the downregulated genes. (D) The mRNA expressions of Map1lc3b, Becn1, Sqstm1, Ulk1, Atg3, Atg4b, Atg5, Atg7, Gabarap, Atg9a, Atg10 and Atg12 of ectopic lesion ( $n=8)$ treated with or without SCM-198. (E, F) The protein expressions of LC3B-II/区, BECN1, ERa and PR in ectopic lesions were measured via Western blotting. (G) The expressions of ERa and PR in ectopic lesion were analyzed by IHC. Data are the presented as the mean \pm SD ( ${ }^{\star \star \star} P<0.001$, compared with EMS group; ns, not significant). 
A

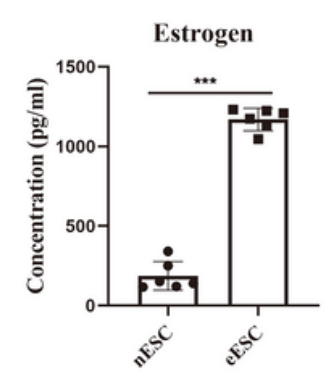

$\mathrm{E}$ nESC1 nESC2 nESC3 eESC1 eESC2 eESC3 kDa

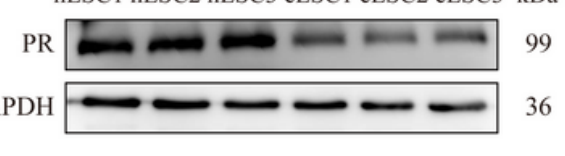

$\mathrm{B}$

nESC1 nESC2 nESC3 eESC1 eESC2 eESC3 kDa

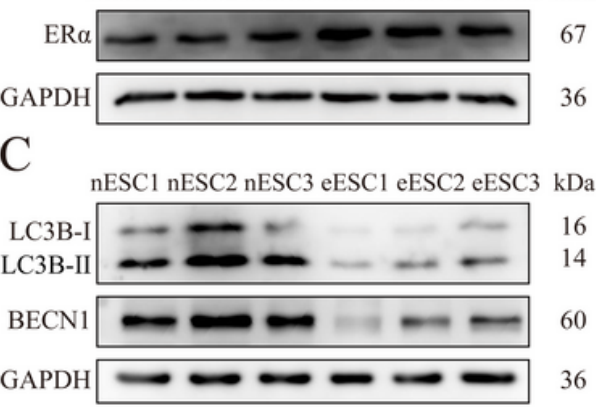

$\mathrm{F}$

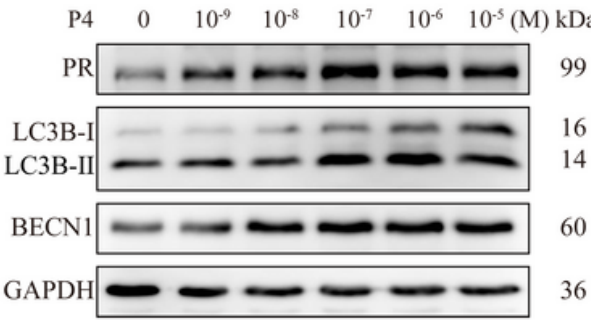

$\mathrm{D}$

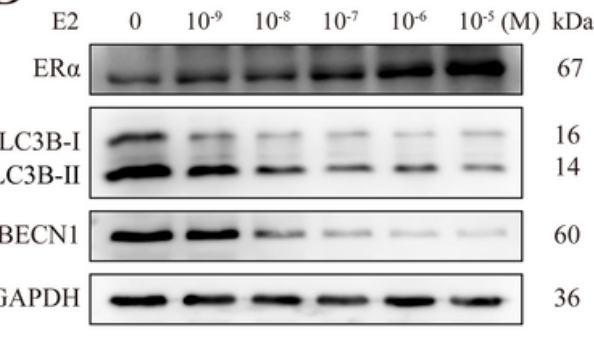

G Ctrl siPR-1 siPR-2 siPR-3 kDa

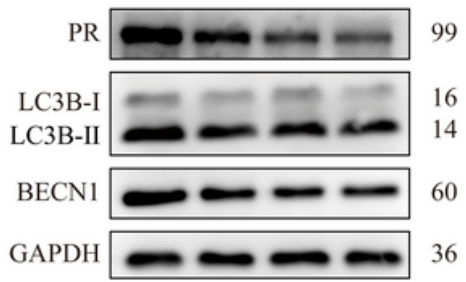

\section{Figure 3}

The ERa/PR imbalance contributes to the hypo-autophagy state of eESCs.

(A) ELISA was utilized to detect the estrogen concentrations of eESCs and nESCs $(n=6)$, both of which

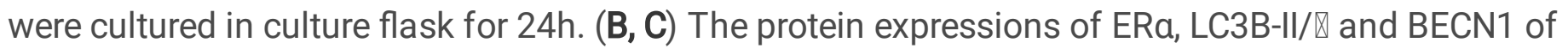
eESCs and nESCs were analyzed by western blotting. (D) After treated with different concentrations of E2

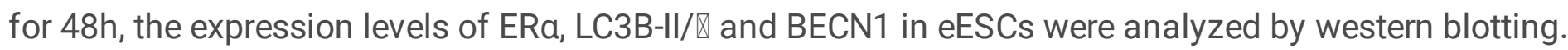
(E) The protein expressions of PR in eESCs and nESCs were detected by western blotting. (F) After treated

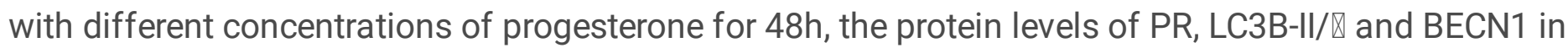

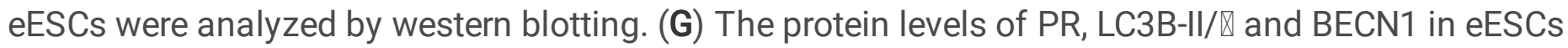
after PR silencing were analyzed by western blotting. Data are the presented as the mean \pm SD $(\star \star \star P<$ $0.001)$. 
A

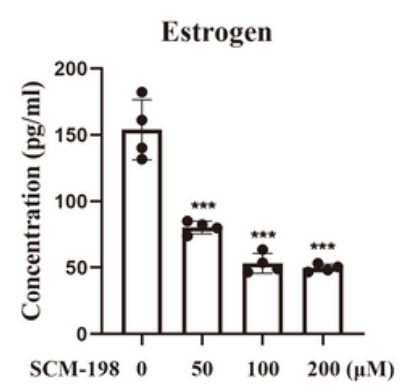

$\mathrm{D}$

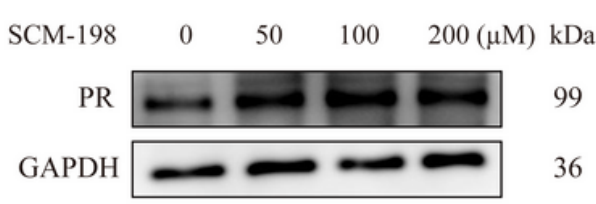

B

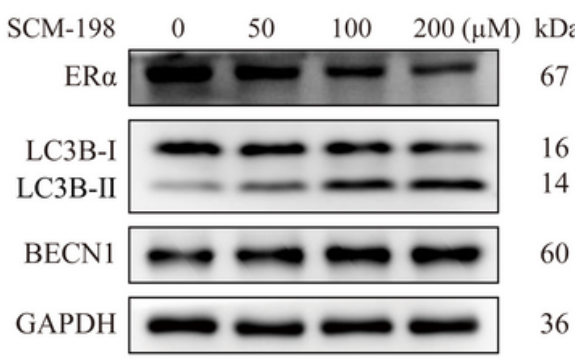

$\mathrm{E}$

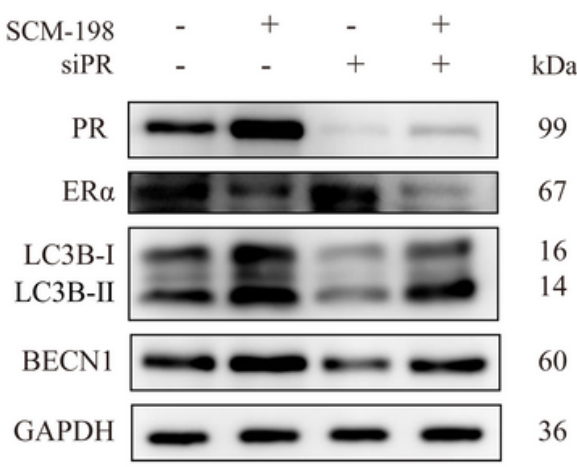

$\mathrm{C}$

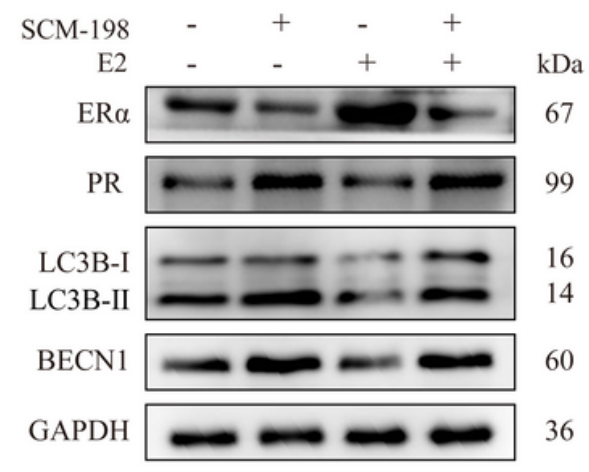

$\mathrm{F}$

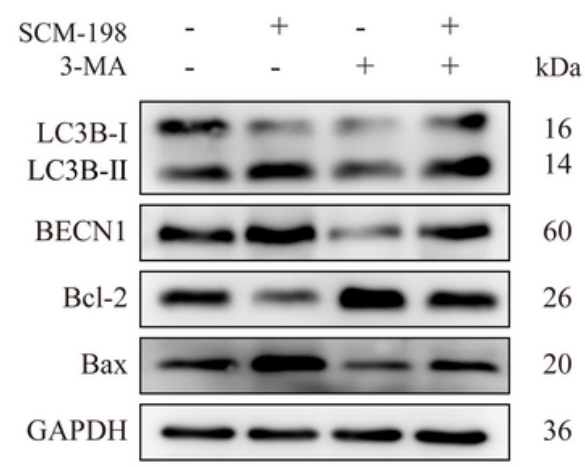

Figure 4

SCM-198 promotes autophagy mediated apoptosis of eESC by inhibiting estrogen-ERa pathway and promoting PR expression.

(A) After treated with different concentrations of SCM-198 for 48h, estrogen concentration of eESCs was analyzed by ELISA $(n=4)$. (B) The eESCs were treated with different concentrations of SCM-198 for 48h,

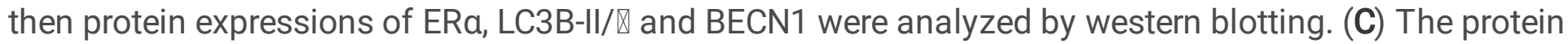
expressions of ERa, PR, LC3B-II/ $\triangle$ and BECN1 were detected by western blotting in eESCs treated with SCM-198 $(200 \mu M)$, E2 $(100 \mathrm{nM})$ or SCM-198+E2 $(200 \mu \mathrm{M}, 100 \mathrm{nM})$ for 48h. (D) The eESCs were treated with different concentrations of SCM-198 for 48h, then protein expression of PR was analyzed by western blotting. (E) After treated with SCM-198 $(200 \mu \mathrm{M})$, silenced PR or SCM-198+ silenced PR for 48h, the

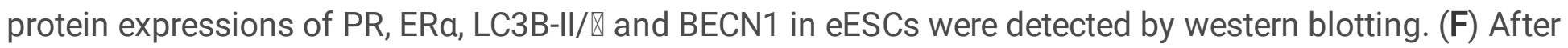
treated with SCM-198 $(200 \mu \mathrm{M})$, 3-MA $(5 \mathrm{mM})$ or SCM-198+3-MA $(200 \mu \mathrm{M}, 5 \mathrm{mM})$ for $48 \mathrm{~h}$, the expressions of LC3B-II/ $\mathbb{B}, \mathrm{BECN} 1, \mathrm{BCl}-2$ and Bax in eESCs were analyzed by western blotting. Data are the presented as the mean $\pm \mathrm{SD}$ (*** $P<0.001$; compared with eESCs that were not treated with SCM-198). 
A

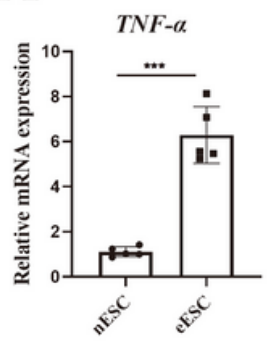

$\mathrm{C}$

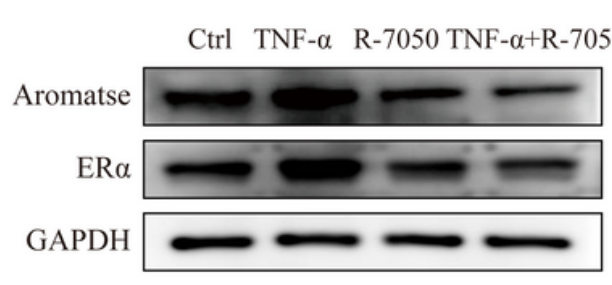

$\mathrm{B}$

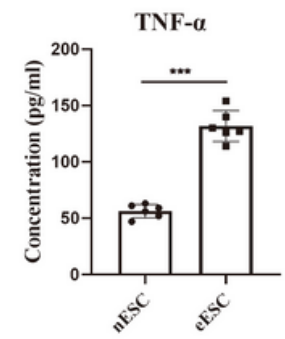

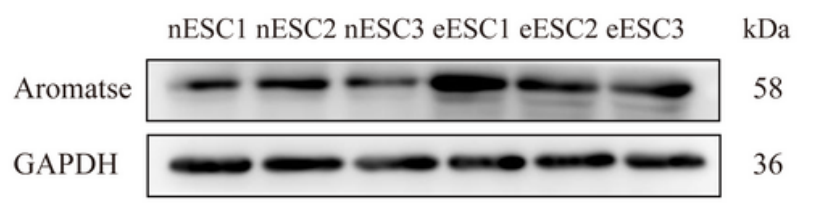

$\mathrm{E}$
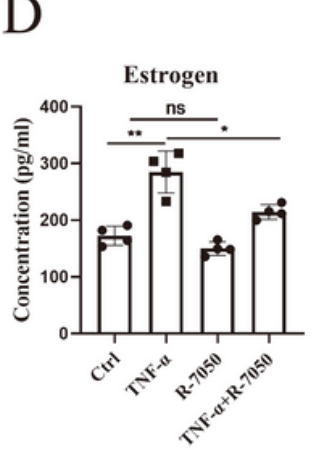

I
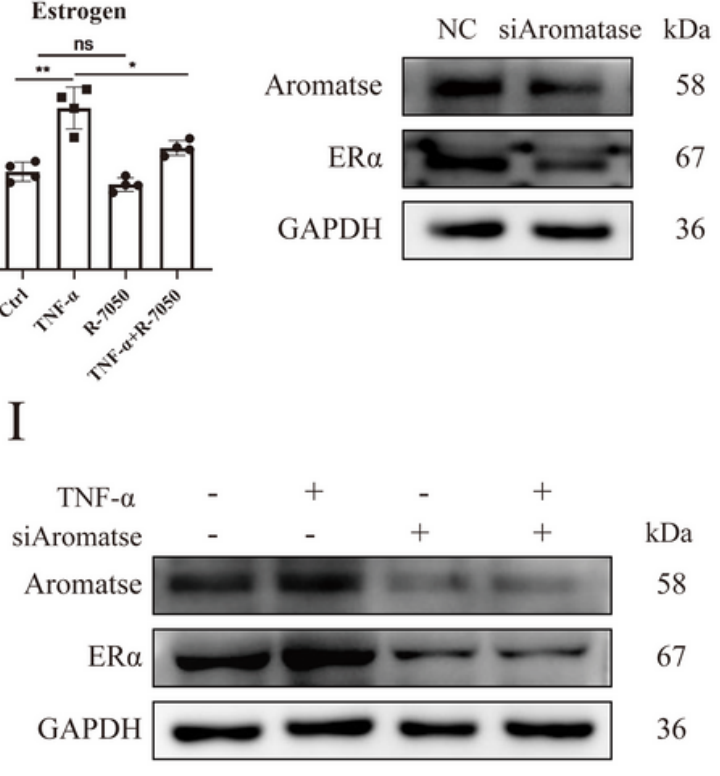

$\mathrm{F}$

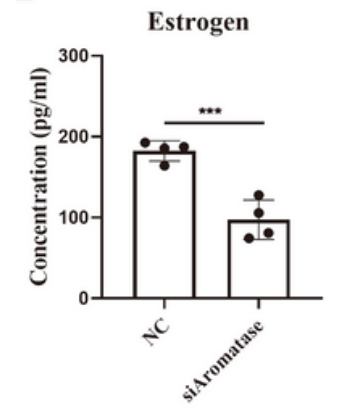

$\mathrm{J}$
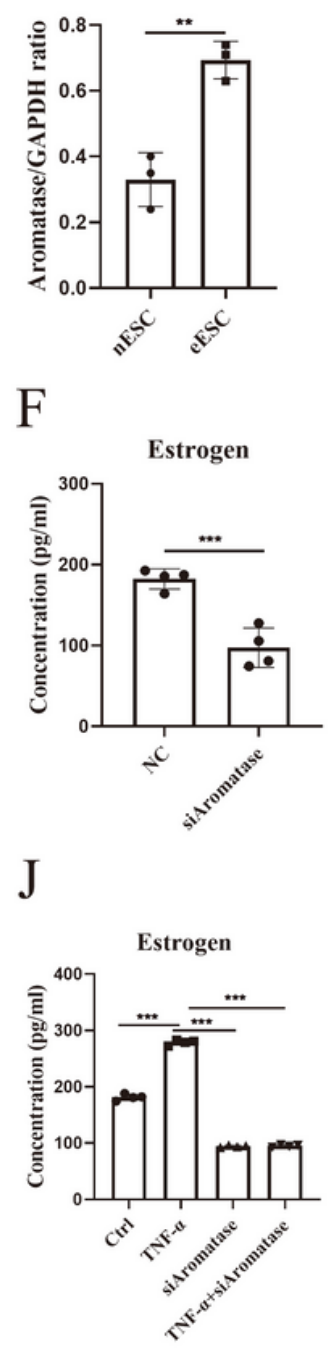

Figure 5

TNF-a upregulates aromatase estrogen-ERa pathway in eESCs.

(A) The mRNA expression $(n=5)$ and concentration $(n=6)$ of TNF- $a$ in eESCs and nESCs were detected by RT-PCR and ELISA. (B) The aromatase expression of eESCs and nESCs were detected by western blotting

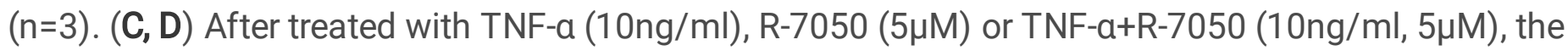
expressions of aromatase and ERa in eESCs were analyzed by western blotting (C) and the concentration of estrogen was detected by ELISA $(n=4)(D)$. $(E-H)$ The aromatase was silenced or overexpressed in eESCs for $48 \mathrm{~h}$. Then, the expressions of aromatase and ERa were detected by western blotting $(\mathbf{E}, \mathbf{G})$ and the level of estrogen was assayed by ELISA $(n=4)(F, H)$. $(I, J)$ The protein expressions of aromatase and ERa were analyzed by western blotting $(\mathrm{I})$ and the concentration of estrogen was detected by ELISA $(\mathrm{n}=4)$ (J). Data are the presented as the mean \pm SD $\left({ }^{\star} P<0.05\right.$, ${ }^{\star *} P<0.01$, and ${ }^{\star * \star} P<0.001$; ns, not significant). 
Figure 6

The pro-apoptotic effects of SCM-198 are realized by downregulating aromataseestrogen pathway via inhibiting TNF-a.

(A) ELISA was utilized to detect TNF-a concentration of eESCs treated with different dose of SCM-198 $(n=4)$. (B) The protein expressions of aromatase and ERa in eESCs treated with different dose of SCM198 were measured by western blotting. (C, D) After treated with TNF-a $(10 \mathrm{ng} / \mathrm{ml}), \mathrm{SCM}-198(200 \mu \mathrm{M})$, or

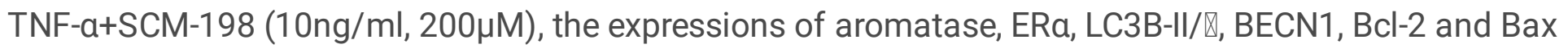
were analyzed by western blotting $(\mathrm{C})$ and the concentration of estrogen concentration was detected by ELISA $(n=4)(D)$. Data are presented as the mean \pm SD $(* \star P<0.01$, and $* \star * P<0.001)$.

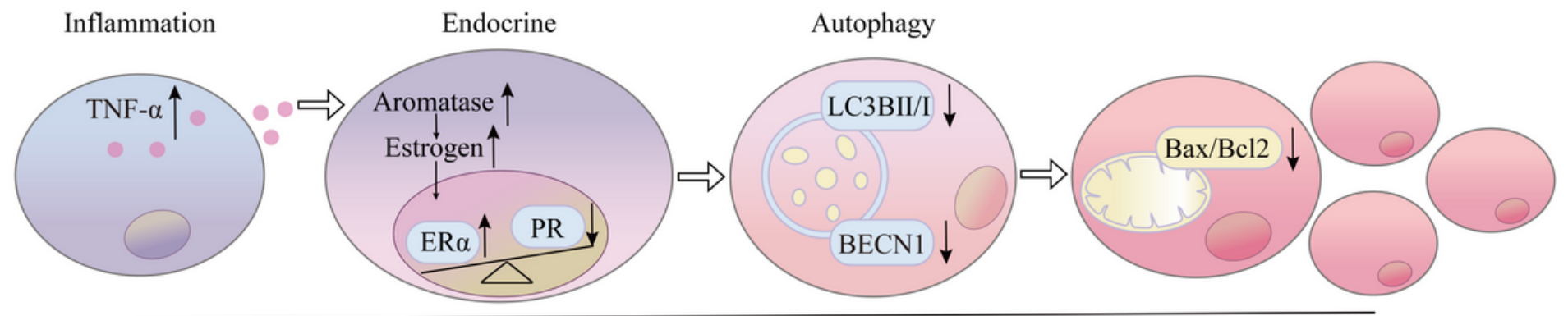

Endometriosis

eESC

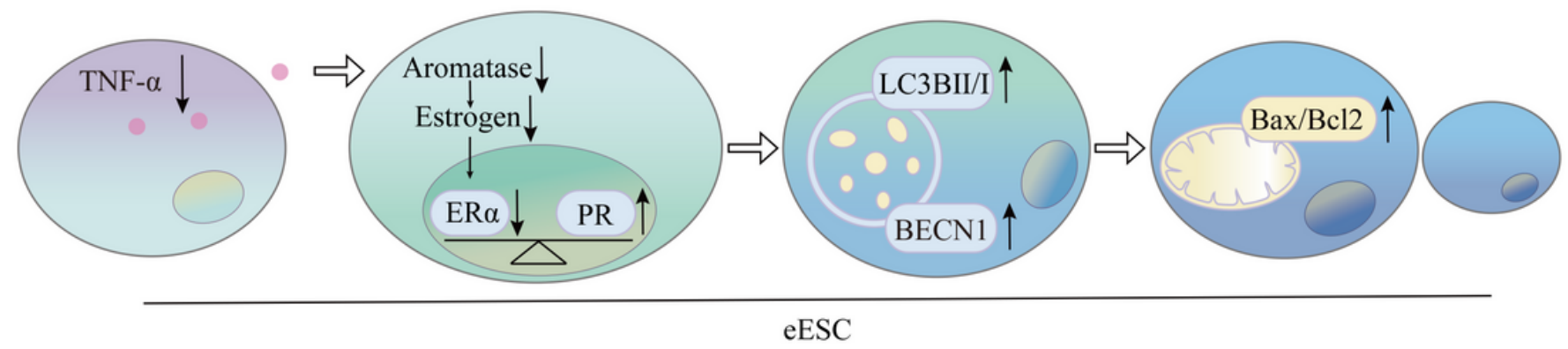

Endometriosis with SCM-198 treatment

Figure 7

Schematic diagram showing therapeutic mechanism of SCM-198 on EMS.

Compared with nESCs, the production of TNF-a was higher in eESCs. Elevated TNF-a augmented the activation of aromatase-estrogen-ERa signaling. The upregulated estrogen signaling and downregulated progesterone signaling co-suppressed the autophagy level, which further conduced to the growth of eESCs. SCM-198 inhibited TNF-a-aromatase-estrogen- ERa signaling and increased PR expression. Consequently, SCM-198 promoted autophagy-mediated apoptosis of eESCs by reconstructing the balance of estrogen and progesterone signals. 